\title{
Cardiovascular risk assessment in rheumatoid arthritis - controversies and the new approach
}

\author{
Krzysztof Bonek, Piotr Głuszko \\ Department of Rheumatology, National Institute of Geriatrics, Rheumatology and Rehabilitation, Warsaw, Poland
}

\begin{abstract}
The current methods of cardiovascular (CV) risk assessment in the course of inflammatory connective tissue diseases are a subject of considerable controversy. Comparing different methods of CV risk assessment in current rheumatoid arthritis (RA) guidelines, only a few of them recommend the use of formal risk calculators. These are the EULAR guidelines suggesting the use of SCORE and the British Society for Rheumatology guidelines performed in collaboration with NICE preferring the use of QRISK-2. Analyzing the latest American and British reports, two main concepts could be identified. The first one is to focus on risk calculators developed for the general population taking into account RA, and the calculator that might fulfill this role is the new QRISK-2 presented by NICE in 2014. The second concept is to create RA-specific risk calculators, such as the Expanded Cardiovascular Risk Prediction Score for RA. In this review we also discuss the efficiency of a new Pooled Cohort Equation and other calculators in the general and RA population.
\end{abstract}

Key words: rheumatoid arthritis, cardiovascular risk calculators, current guidelines.

\section{Introduction}

Relying on multiple sources, it could be concluded that overall cardiovascular risk in rheumatoid arthritis (RA) patients has two components. The first one is a set of traditional risk factors for the general population. The INTERHEART [1] study has demonstrated the effect of the well-known factors associated with atherosclerosis: abdominal obesity, smoking status, hypertension, diabetes, lipid disturbances, psychosocial factors and lifestyle. The second component is a set of risk factors such as inflammatory mediators, treatment and disease activity in RA [2].

Despite the fact that the Framingham Heart Study [3] has shown a positive correlation of high-density lipoprotein $(\mathrm{HDL})$ levels in relation to cardiovascular (CV) risk reduction, more recent findings revealed the effect of inflammation on the formation of "dysfunctional HDL" which lacks a CV disease preventive function [4, 5]. Moreover, there are reports indicating that lipoprotein(a) [6] and apolipoprotein B (Apo-B) [7] and homocysteine [8] metabolism might be altered due to RA progression. RA progression related factors such as time-av- eraged disease activity [9], high activity expressed as the time-averaged DAS28 [10], the Simplified Disease Activity Index (SDAI), the Clinical Disease Activity Index (CDAI) [11], C-reactive protein (CRP) [12-14] levels or seropositivity [14] influence the $\mathrm{CV}$ risk. The influence of RA treatment on CV risk is not quite clear. The QUEST-RA and other studies have shown reduction of cardiovascular events when applying methotrexate (MTX) [15-18] and sulfasalazine (SLF) [15]. In the case of disease-modifying anti-rheumatic drugs (DMARDs), the influence on cardiovascular risk remains controversial. Solomon et al. [19] postulated a possible increase of CV risk when applying leflunomide, cyclosporine and azathioprine, while other authors found a beneficial influence of cyclosporine [17] and leflunomide [18] on CV risk. Cardiovascular risk reduction has not been proven for either gold salts or antimalarial medications [15]. The effect of biological drugs on CV events remains controversial as well. In the QUEST-RA study, it was suggested that anti-tumor necrosis factor (anti-TNF) antibodies may reduce cardiovascular risk [15]. However, other authors do not share

\footnotetext{
Address for correspondence:

Krzysztof Bonek, Department of Rheumatology, National Institute of Geriatrics, Rheumatology and Rehabilitation, Spartańska 1,

02-637 Warsaw, Poland, e-mail: krzysztof.bonek@gmail.com
}

Submitted: 25.05.2016, Accepted: 21.06.2016 
that view unanimously $[17,18]$. Observations made by some investigators suggest that patients with hypertension treated with biological agents had twice the risk of cardiovascular events, whereas risk reduction could be associated with the simultaneous use of methotrexate [20]. Another factor is presumed corticosteroid intake and its role in the treatment [21, 22]. In a recent meta-analysis the increased CV risk due to corticosteroid uptake was highlighted [23]. The use of nonsteroidal anti-inflammatory drugs (NSAIDs) seems to be associated with increased CV risk [24], but it requires further research as there are opinions that NSAIDs might lower the CV risk in RA [25]. In any case, the chronic use of NSAIDs should be reevaluated.

Recently, a EULAR working group led by Nurmohamed [26] proposed 11 guidelines for cardiovascular risk assessment for patients with RA, ankylosing spondylitis (AS) and psoriatic arthritis (PsA). They also pointed out the need to create a new RA-focused cardiovascular risk calculator, stating that the current methods of cardiovascular risk assessment are maladjusted [26]. Briefly, the following guidelines were proposed:

1. Rheumatoid arthritis and ankylosing spondylitis are associated with higher cardiovascular risk. This is due to the "traditional" risk factors and active inflammation. Occurrence of cardiovascular events in psoriatic arthritis might be increased.

2. Active inflammation is associated with increased cardiovascular risk in patients with RA, AS and PSA. To reduce the risk, it is necessary to reduce disease activity.

3. In each patient with RA, AS and PsA, cardiovascular risk should be assessed every 5 years and after all treatment modifications.

4. It is recommended to use the national guidelines for cardiovascular risk assessment in RA, AS and PsA. If no such guidelines exist, it is recommended to use the Systematic Coronary Risk Evaluation (SCORE) equation.

5. In cardiovascular risk assessment of patients with RA, AS and PSA, the ratio of total cholesterol (TC) and high-density lipoprotein (HDL) levels should be applied. Lipid parameters should be tested in remission or stable disease activity.

6. If RA is not a parameter taken into account by a given risk calculator, after fulfillment of the EULAR criteria [presence of anti-cyclic citrullinated peptide antibodies (anti-CCP) or positive rheumatoid factor $(R F)$, disease duration of over 10 years, extra-articular symptoms], the final score should be the risk score multiplied by a coefficient of 1.5 .

7. Only credible risk calculators approved by relevant national associations should be applied. Statin ther- apy and antihypertensive treatment should depend on guidelines of relevant national associations.

8. Special caution should be taken when applying NSAIDs in patients with RA and PSA with documented cardiovascular diseases, or when cardiovascular risk factors coexist. NSAIDs should be used as first line treatment in AS unless contraindications coexist. 9. In chronic treatment, the lowest possible dose of corticosteroids should be applied. The objective should be to reduce corticosteroid intake after achieving low disease activity. Indications for application of corticosteroids should be reviewed on a regular basis.

10. When recommending to change an individual's lifestyle, it is necessary to focus on a healthy diet, regular physical activity, and smoking cessation.

11. When conducting screening for subclinical atherosclerosis, carotid artery ultrasound may be advisable. Comparing the EULAR guidelines of 2010 with the new proposal of 2015, some differences can be found. A statement was made that CV risk in RA is associated with a higher incidence of population risk factors and inflammation. Higher occurrence of traditional risk factors increases cardiovascular risk in AS. Moreover, a recommendation to assess CV risk every 5 years was added. Nurmohamed's group have pointed out that PSA and AS are associated with increased cardiovascular risk, whereas in the guidelines of 2010, the above statement was forwarded only as a conjecture. Another new recommendation is the use of carotid ultrasound in the search for atherosclerotic plaques; however, not all experts share this view. Carotid intima-media complex (IMC) thickening has been found in RA patients without clinical determinants of atherosclerosis [27]. Yet, there are no clear guidelines as to the frequency of records and the period of disease progression within which to start to keep records. Lastly, attention was paid to the fact that the effect of NSAIDs on cardiovascular risk requires further studies. There are two notable modifications implemented to the cardiovascular risk assessment using the CV risk calculator. As SCORE and Framingham Risk Score (FRS) were created for the general population, the EULAR experts recommend multiplying cardiovascular risk in RA patients by 1.5 if patients have two out of three factors: anti-CCP or RF positivity or RA progression over 10 years or presence of extra-articular symptoms [28]. This coefficient, based on assessment of risk of death of patients with RA compared with the general population risk [29], is widely criticized, pointing to the ineffectiveness of the above modification to cardiovascular risk assessment [30, 31].

The SCORE calculator was developed in 1994 by the European Society of Cardiology (ESC) and the European Society of Hypertension (ESH) [32]. This is a key tool used 
for cardiovascular risk assessment in European countries. Many societies recommended the use of the SCORE equation for cardiovascular risk assessment in 2012 [32]. Advantages of the SCORE calculator refer to clinical evidence supported by studies performed in 12 European cohorts (over 700,000 patients), and simplicity of its application. It calculates 10-year cardiovascular mortality risk. The populations to be assessed are people aged 40 and older in the primary prevention of cardiovascular events. For people under 40, relative risk charts were created. The parameters taken into account by this calculator include gender, age, smoking status, systolic blood pressure, total cholesterol and BMI. On the basis of calculated scores, patients are classified into four risk groups: < 1\%; 1-4\%; $5-9 \% ; \geq 10 \%$. In each of the risk groups, there are the socalled primary (low-density lipoprotein (LDL)) level) and the secondary (non-high-density-lipoprotein (non-HDL) cholesterol and lipoprotein (a) (LPA) targets, known as therapeutic targets [32].

There are some limitations with the application of the SCORE equation:

1. The SCORE equation is only a part of the algorithm applied to classify patients into particular risk groups [32]. There are conditions for which higher cardiovascular risk has been observed compared to the calculations obtained with the SCORE calculator, such as chronic kidney disease or diabetes mellitus. Although RA is a factor of its importance comparable to the CV risk caused by type 2 diabetes, RA has not been included in the algorithm [33].

2. The SCORE equation is used to assess cardiovascular mortality risk and does not take into account non-fatal cardiovascular events. According to the European Society of Cardiology and European Atherosclerotic Association (ESC/EAS) experts, the score obtained using the SCORE equation for all potential cardiovascular events (fatal and non-fatal) should be multiplied by 3 [32]. For example, 10\% SCORE risk is equal to 30\% risk of a non-fatal CV event.

3. There are many groups of patients whose scores, regardless of the presence of RA, will be unreliable. These are patients entering into a higher age group, according to the SCORE equation, as well as patients with cardiovascular mortality risk factors due to their family history. In addition, the SCORE equation does not include the so-called residual risk that results from as yet undiscovered risk factors

4. Application of the SCORE equation may cause various difficulties in evaluation of RA patients because it does not recognize validated cardiovascular risk factors, such as disease progression, high clinical activity, higher CRP levels and seropositivity [21]. Experts, under the direction of E.E.A. Arts, have advised modification of the SCORE algorithm by adding the following factors: age, gender, seropositivity, presence of anti-CCP antibodies, DAS28, ESR, and HAQ [30]. Unfortunately the algorithm modified by adding the above factors did not result in any improvement in cardiovascular risk assessment [30].

5. The ESC/EAS experts introduced the table of interventions [32] depending on whether a patient has achieved the LDL target level, dependent on a given risk group. Pharmacotherapy therefore became conditional upon (even in some high and very high risk groups) achieving a specific LDL level, which is the primary therapeutic target according to the ESC/EAS. In patients achieving recommended LDL cholesterol levels in whom it is advisable to further reduce the risk, it is necessary to consider the secondary targets which are specific non-HDL cholesterol levels and LPA. In RA, the table of interventions seems to be misleading, since RA patients present lipid disturbances caused by active inflammatory processes [34].

6. Comparative research has shown that the SCORE equation and its modification by the EULAR experts are insufficient to indicate precisely the populations requiring risk reduction. In the study led by Arts [35], four risk equations were compared (FHS, SCORE, RRS, QRISK-2), proving that none of the algorithms was able to correctly classify patients into specific risk groups.

7. It also appeared that the EULAR modification called the mSCORE (1.5 coefficient applied after meeting the above criteria) underestimated the risk in low- and moderate risk patients, while most of the RA patients were classified into these two groups. Furthermore, the mSCORE overestimated the risk in high-risk patients [31].

In everyday practice a number of patients with RA do not receive adequate treatment regardless of developed atherosclerosis. Studies which correlated the recommended SCORE calculator with records of carotid intima-media thickness have proven the presence of atherosclerotic plaques already in patients from moderate-risk groups [36]. According to the ESC/EHA guidelines, these patients should be excluded from the SCORE evaluation, proving in such situations a very high cardiovascular disease risk. Carotid intima-media thickening (IMC) has been found in RA patients without clinical determinants of atherosclerosis [27]. Despite the existence of the EULAR guidelines for the use of carotid ultrasound in risk assessment [26], there are no clear guidelines as to the frequency of records and the period of disease progression within which to start to keep records. In our opinion [37] and according to other experts [36, 38], the usefulness of this non-invasive test for cardiovascular risk assessment has been confirmed. 
The QRISK-2 calculator is the most recent version of the English and Welsh risk prediction algorithm created on the basis of reports of primary care physicians [39]. From 2014, this calculator became an essential tool in assessing overall cardiovascular risk in the UK, replacing the Framingham scoring. The QRISK-2 calculator is the only calculator which in the equation takes into account RA as a separate cardiovascular risk factor. It calculates the percentage of the risk of cardiovascular disease in the populations between 24 and 84 years of age. It takes into account ethnicity, age, smoking status, gender, systolic blood pressure, total cholesterol, HDL cholesterol, BMI, family history, zip code, antihypertensive treatment, rheumatoid arthritis, chronic kidney disease, diabetes, and atrial fibrillation [39]. Pharmacological intervention (application of statins) is recommended in patients with the threshold of $20 \%$ of the end event over the following 10 years [39]. The QRISK-2 calculator cannot be recommended for other countries because the calculator corresponds to the populations in England and Wales. An interesting aspect of the QRISK-2 equation is the use of a risk modifier of 1.4. In practice, the authors used the EULAR modification for all RA patients. The calculator might increase the number of patients eligible for statin therapy (those with risk between 14\% and $20 \%$ excluding RA). This might allow the problem of underestimation in risk groups to be solved (especially low- and moderate cardiovascular risk), but on the other hand, some patients will be exposed to the side effects of statins and will receive recommendations to use a drug potentially not improving the prognosis. The effectiveness of the QRISK-2 calculator in RA needs more extensive evaluation.

It is necessary to consider the new guidelines presented in the USA in 2013 by the American College of Cardiology/American Heart Association (ACC/AHA) [40]. Their CV risk estimation method is based on a new Poled Cohort Equation formula. It was found that implementation of the new guidelines led to many more patients being eligible for statin therapy. In one trial in the ACC/AHA prediction model, $95.6 \%$ of male patients and $65.8 \%$ of female patients over 55 years of age were qualified for statin treatment [41]. In comparative studies with RA patients [42] including the guidelines of the European Society of Cardiology (ESC) (the SCORE), including modifications with the coefficient of 1.5 according to the EULAR, the Adult Treatment Panel III (ATP-III) (the Framingham Risk Score) and the ACC/AHA (the Pooled Cohort Equations) have shown that statin therapy was recommended in $9.6 \%$ of the women and $26.1 \%$ of the men according to the SCORE algorithm, whereas according to the ATP-III guidelines the inclusion of statins was recommended in $15.5 \%$ of the women and $51 \%$ of the men. According to the ACC/AHA equation, $78.5 \%$ of the men and $38.8 \%$ of the women should be treated with statins. The investigators stated that there is a tendency to classify RA patients in the high risk groups using the ACC/AHA guidelines [41]. Their data suggest that, following ACC/AHA guidelines, the majority, if not all, RA patients would be treated with statins. Despite its potential for CV risk overestimation [43], it is considered to be an effective method for CV risk calculation in the US population [44].

Special attention should be paid to the most recent cardiovascular risk calculator, called the Expanded Cardiovascular Risk Prediction Score for Rheumatoid Arthritis (ERS-RA). This calculator was based on the CORRONA registry, a cohort of 23,605 patients with RA in the primary prevention of cardiovascular events. For 2.2 years, patients were analyzed in terms of the occurrence of end points including myocardial infarction, ischemic stroke and cardiovascular related death. Based on the analysis of all considered variables, two groups of risk factors in RA patients were distinguished, and then included in the calculator. The first group consisted of the "traditional" risk factors such as age, gender, type 2 diabetes, hyperlipidemia, hypertension, and smoking status. The second group included factors that impact the RA-specific cardiovascular risk such as clinical activity of the disease expressed as the Clinical Disease Activity Index (CDAI), the degree of physical fitness, expressed as the Health Assessment Questionnaire Disability Index (M-HAQ-DI), corticosteroid intake over $10 \mathrm{mg}$ prednisone per day and disease duration of over 10 years [45]. Introduction of the ERS-RA raises some questions and comments: It is unclear whether the calculator developed for the US population will turn out to be sufficient in the evaluation of other countries' populations. Moreover, due to a relatively short time of follow-up, the authors extrapolated the results to the 10-year risk. It seems that further research will be necessary to assess the effectiveness of the calculator in patients with early rheumatoid arthritis and in patients with long disease duration. In addition, imaging diagnostics were not used in screening for atherosclerosis, which could hypothetically change the qualification of some patients to the specific risk groups.

The Reynolds Risk Score is a risk calculator which takes into account the "traditional" risk factors and a history of myocardial ischemia in a patient's family, and high sensitivity C-reactive protein (hs-CRP) levels in women, based on two cohorts of nearly 25,000 women [46]. The calculator was later adapted to the male population and then evaluated in a study on a cohort of over 10,000 men [47]. The assessed factors include: age (3 age categories), total cholesterol levels, HDL cholesterol lev- 
Table I. "Hard" and "general" evidence of CVD in FRS calculator

\begin{tabular}{|ll|}
\hline $\begin{array}{l}\text { "Hard" evidence of } \\
\text { cardiovascular disease }\end{array}$ & $\begin{array}{l}\text { “General" evidence of } \\
\text { cardiovascular disease }\end{array}$ \\
\hline $\begin{array}{l}\text { cardiac death, stroke, } \\
\text { myocardial infarction }\end{array}$ & $\begin{array}{l}\text { cardiac death, myocardial } \\
\text { infarction, heart failure, overt } \\
\text { coronary heart disease, angina } \\
\text { pectoris, ischemic stroke, } \\
\text { hemorrhagic stroke and transient } \\
\text { ischemic attack (TIA) }\end{array}$ \\
\hline
\end{tabular}

els, systolic blood pressure, active cigarette smoking, myocardial infarction in family members under 60 years of age, hs-CRP levels, and glycosylated hemoglobin levels (in the equation for men, this factor was eliminated) $[46,47]$. When assessing the CV risk with this calculator, attention should be paid to the following facts:

- Evaluation of hs-CRP levels in patients with systemic inflammatory diseases will not be credible since CRP level rises with disease activity;

- In comparative studies, the Reynolds equation underestimated cardiovascular risk in patients with RA [35];

- Lastly, this calculator is not recommended by the Polish Cardiac Society [32] nor by a number of other European expert groups.

The Framingham Heart Score (FRS) was published in 1998 owing to large cohort studies carried out in the town of Framingham [48]. There are two variants of this simple diagnostic tool. The first variant estimates the 30-year risk with its endpoint related to the so-called "hard" evidence of cardiovascular disease and "general" evidence of cardiovascular disease (Table I). The evaluated populations are people between 20 and 59 years of age without overt cardiovascular disease or cancer. The assessed factors include gender, age, systolic blood pressure, antihypertensive treatment, smoking status, type 2 diabetes, total cholesterol level and level of HDL or BMI in a simplified model [48]. The second variant of the calculator is used to evaluate the 10-year risk of the end event. Those end events are defined as "general" evidence of cardiovascular disease (Table I). The evaluated populations are people between 30 and 74 years of age without overt cardiovascular disease. The assessed factors are age, diabetes, smoking, antihypertensive treatment, level of total cholesterol, level of HDL cholesterol or BMI in the case of the simplified model [47]. In both variants patients are classified into the following risk groups: < $10 \%$, between 10 and $20 \%$, and over $20 \%$ with the end event.

Introduction of the FRS was potentially a very useful solution based on reliable research and assessment of overall risk of cardiovascular events. The FRS refers to patients aged 30 and older. Moreover, the 30-year risk version offers long-term CV risk assessment. Regarding RA, this equation in the studies of Arts [30] and some other studies [31, 50] did not allow for proper assessment of RA-specific cardiovascular risk. The application of this calculator is associated with a number of limitations. First of all, the FRS, like the SCORE, takes into account neither clinical activity of the disease nor lipid disturbances typical for active RA. There are also studies remarking lower accuracy of risk assessment in young patients, especially in women (with predominant RA) [51] and systemic inflammatory diseases [52]. In FRS trials with EULAR modifications (mFRS), most patients with RA were classified into a low-risk group ( $<10 \%)$, despite proven high "calcium scores" [50], which means that it underestimated the risk for patients with RA.

The PROCAM calculator was developed as a follow-up to the Prospective Munster Heart Study (PROCAM) [53]. This study was started in 1971 to analyze CV risk factors in over 20000 employees in Germany, The PROCAM calculator is used to assess the 10-year risk of heart attack (fatal and non-fatal) and sudden death. The assessed populations were over 7000 working men between 35 and 65 years of age. The factors taken into account in the study included age, smoking status, levels of HDL, LDL, triglycerides, diabetes mellitus, family history, and systolic blood pressure [54]. Compared to the previous calculators, the PROCAM calculator takes into account more metabolic parameters such as lipids and glucose levels. Available studies demonstrate the lipid paradox in RA, where low levels of total cholesterol (TC), LDL and a lower atherogenic index correlate with higher cardiovascular risk [34]. The paradox virtually disqualifies the calculator for RA patients. It has been proven in the study carried out by a team of French, German and Romanian [31] investigators that the PROCAM calculator did not demonstrate any advantage over the SCORE [31]. In addition, the PROCAM calculator was based on a study of a male population, which significantly complicates risk assessment in the population of patients with RA, where women constitute the majority.

Currently a new calculator for RA patients called ATACC-RA [55] is being developed, using a new mathematical model based on nearly 8 years of observation of a cohort of 3176 patients in the primary prevention of cardiovascular events. The ATACC-RA will take into account age, gender, active cigarette smoking, hypertension, the ratio of TC/HDL, seropositivity and DAS28 [55]. This calculator is in the process of evaluation.

Noteworthy is another approach recommended by a group of Dutch experts in risk assessment: simply, 15 years should be added to the age of each RA patient [56]. However, there has been no research done on this approach in relation to populations other than Dutch. 


\section{Summary}

As current methods seem to be maladjusted, there is a need to create a new algorithm for CV risk assessment for patients with rheumatoid arthritis and other inflammatory rheumatic diseases. So far, we have not reached a consensus between different societies on CV risk management in RA. In our opinion it is necessary to reach an agreement between rheumatologists, cardiologists and general practitioners for screening of CV diseases in patients with RA. Future comparative studies on two major attempts, an RA specific calculator (ERS-RA) vs a calculator for the general population including RA (QRISK-2), might bring a conclusion on the general concept of CV risk assessment. Another approach is an active search for atherosclerotic plaques. It has been confirmed that atherosclerosis develops in patients with RA earlier compared to the general population [14]. Following 2015 EULAR guidelines [26], it might be useful to use ultrasound of carotid arteries for this task. However, it has not been specified how often and when to start testing. It is a simple, widely available imaging diagnostics tool bearing practically no risk for patients. In our opinion [36], and following Gonzáles-Gay et al. [38], ultrasound of carotid arteries could be recommended to the majority of RA patients, especially in patients classified in the high CV risk group and with extra-articular manifestations and RF or anti-CCP antibodies positivity or 10-year disease progression. During the EULAR 2016 session Sattar [57] suggested that the best way to assess the CVD risk in the individual patient is just to follow recent EULAR guidelines.

The authors declare no conflict of interest.

\section{References}

1. Yusuf S, Hawken S, Ounpuu S. Effect of potentially modifiable risk factors associated with myocardial infarction in 52 countries (the INTERHEART study): case-control study. Lancet 2004; 364: 937-945

2. Nurmohamed M, Herslinga M, Kitas GD. Cardiovascular comorbidity in rheumatic diseases. Nat Rev Rheumatol 2015; 11: 693-704.

3. Wilson P, Abbott R, Castelli W. High density lipoprotein cholesterol and mortality. The Framingham Heart Study. Arteriosclerosis 1988; 8: 737-741.

4. Smith JD. Myeloperoxidase, inflammation, and dysfunctional HDL. J Clin Lipidol 2010; 5: 382-388.

5. Głuszko P, Filipowicz-Sosnowska A, Rupiński R. Lipid profile in rheumatoid arthritis patients treated with disease-modifying antirheumatic drugs. Reumatologia 2014; 52: 120-128.

6. Dursunoğlu D, Evrengül H, Polat B. Lipoprotein and lipids in patients with rheumatoid arthritis: serum levels and relationship to inflammation. Rheumatol Int 2005; 25: 241-245.
7. Öhman M, Öhman ML, Wållberg-Jonsson. The apoB/apoA1 ratio predicts future cardiovascular events in patients with rheumatoid arthritis. Scand J Rheumatol 2014; 43: 259-264.

8. Sattar N, McCarey DW, Capell H. Explaining how "high-grade" systemic inflammation accelerates vascular risk in rheumatoid arthritis. Circulation 2003; 108: 2957-2963.

9. Solomon DH, Reed GW, Kremer JM, et al. Disease activity in rheumatoid arthritis and the risk of cardiovascular events. Arthritis Rheum 2015; 67: 1449-1455.

10. Arts EE, Fransen J, den Broeder AA, et al. Extended report The effect of disease duration and disease activity on the risk of cardiovascular disease in rheumatoid arthritis patients. Ann Rheum Dis 2015; 74: 998-1003.

11. Smolen JS, Aletaha D. Scores for all seasons: SDAI and CDAI. Clin Exp Rheumatol 2014; 32: 75-79.

12. Goodson NJ, Symmons DP, Scott DG, et al. Baseline levels of C-reactive protein and prediction of death from cardiovascular disease in patients with inflammatory polyarthritis:a tenyear followup study of a primary care-based inception cohort. Arthritis Rheum 2005; 52: 2293-2299.

13. Navarro-Millán I, Yang S, DuVall SL, et al. Association of hyperlipidaemia, inflammation and serological status and coronary heart disease among patients with rheumatoid arthritis: data from the National Veterans Health Administration. Ann Rheum Dis 2016; 75: 341-347.

14. Goodson N, Marks J, Lunt M, et al. Cardiovascular admissions and mortality in an incepcion cohort of patients with rheumatoid arthritis with onset in the 1980s and 1990s. Ann Rheum Dis 2005; 64: 1595-1601.

15. Naranjo A, Sokka T, Descalzo MA, et al. Cardiovascular disease in patients with rheumatoid arthritis: results from the QUEST-RA study. Arthritis Res Ther 2008; 10: R30.

16. Westlake SL, Colebatch AN, Baird J, et al. The effect of methotrexate on cardiovascular disease in patients with rheumatoid arthritis: a systematic literature review. Rheumatology (Oxford) 2010; 49: 295-307.

17. Kisiel B, Kruszewski R, Juszkiewicz A, et al. Methotrexate, cyclosporine $\mathrm{A}$, and biologics protect against atherosclerosis in rheumatoid arthritis. J Immunol Res 2015; 2015: 759610.

18. Suissa S, Bernatsky S, Hudson M. Antirheumatic drug use and the risk of acute myocardial infarction. Arthritis Rheum 2006; 55: 531-536.

19. Solomon DH, Avorn J, Katz JN, et al. Immunosuppressive medications and hospitalization for cardiovascular events in patients with rheumatoid arthritis. Arthritis Rheum 2006; 54: 3790-3798.

20. Baghdadi LR, Woodman RJ, Shanahan EM, et al. The impact of traditional cardiovascular risk factors on cardiovascular outcomes in patients with rheumatoid arthritis: a systematic review and meta-analysis. PLoS One 2015; 10(2): e0117952.

21. Davis JM, Maradit Kremers H, Crowson CS, et al. Glucocorticoids and cardiovascular events in rheumatoid arthritis: A population-based cohort study. Arthritis Rheum 2007; 56: 820-830.

22. Kaplan MJ. Cardiovascular complications of rheumatoid arthritis - assessment, prevention and treatment. Rheum Dis Clin North Am 2010; 36: 405-426. 
23. Roubille C, Richer V, Starnino T. The effects of tumor necrosis factor inhibitors, methotrexate, non-steroidal anti-inflammatory drugs and corticosteroids on cardiovascular events in rheumatoid arthritis, psoriasis and psoriatic arthritis: a systematic review and meta-analysis. Ann Rheum Dis 2015; 74: 480-489.

24. Głuszko P, Bielińska A. Non-steroidal anti-inflammatory drugs and the risk of cardiovascular diseases: are we going to see the revival of cyclooxygenase-2 selective inhibitors? Pol Arch Med Wewn 2009; 119: 231-235.

25. Lindhardsen J, Gislason GH, Jacobsen S, et al. Non-steroidal anti-inflammatory drugs and risk of cardiovascular disease in patients with rheumatoid arthritis: a nationwide cohort study. Ann Rheum Dis 2014; 73: 1515-1521.

26. Nurmohamed M. Speaker Presentations Eular Recommendation Update on Cardiovascular Disease in RA. Ann Rheum Dis 2015; 74 (suppl 2): 9 Available at pathlms.com/eular/ events/363/thumbnails_video_presentations/13056.

27. van Sijl AM, Peters MJ, Knol DK, et al. Carotid intima media thickness in rheumatoid arthritis as compared to control subjects: a meta-analysis. Semin Arthritis Rheum 2011; 40: 389 397.

28. Turesson C, O'Fallon WM, Crowson CS, et al. Occurrence of extraarticular disease manifestations is associated with excess mortality in a community based cohort of patients with rheumatoid arthritis. J Rheumatol 2002; 29: 62-67.

29. Peters MJ, Symmons DP, McCarey D, et al. Recommendations EULAR evidence-based recommendations for cardiovascular risk management in patients with rheumatoid arthritis and other forms of inflammatory arthritis. Ann Rheum Dis 2010; 69: 325-331.

30. Arts EEA, Popa CD, den Broeder AA, et al. Prediction of cardiovascular risk in rheumatoid arthritis: performance of original and adapted SCORE algorithms. Ann Rheum Dis 2016; 75: 674-680.

31. Purcarea A, Sovaila S, Gheorghe A, et al. Cardiovascular disease risk scores in the current practice: which to use in rheumatoid arthritis? J Med Life 2014; 7: 461-467.

32. Perk J, De Backer G, Gohlke H, et al. European Guidelines on cardiovascular disease prevention in clinical practice (version 2012). The Fifth Joint Task Force of the European Society of Cardiology and Other Societies on Cardiovascular Disease Prevention in Clinical Practice (constituted by representatives of nine societies and by invited experts). Eur Heart J 2012; 33: 1635-1701.

33. Lindhardsen J, Ahlehoff O, Gislason GH, et al. The risk of myocardial infarction in rheumatoid arthritis and diabetes mellitus: a Danish nationwide cohort study. Ann Rheum Dis 2011; 70: 929-934.

34. Myasoedova E, Crowson CS, Kremers HM. Lipid paradox in rheumatoid arthritis: the impact of serum lipid measures and systemic inflammation on the risk of cardiovascular disease. Ann Rheum Dis 2011; 70: 482-487.

35. Arts EEA, Popa C, Broeder AA, et al. Performance of four current risk algorithms in predicting cardiovascular events in patients with early rheumatoid arthritis.. Ann Rheum Dis 2015; 74: 668-674.
36. Corrales A, Parra JA, González-Juanatey C, et al. Cardiovascular risk stratification in rheumatic diseases:carotid ultrasound is more sensitive than Coronary Artery Calcification Score to detect subclinical atherosclerosis in patients with rheumatoid arthritis. Ann Rheum Dis 2013; 72: 1764-1770.

37. Głuszko P, Bonek K. Statins in rheumatology: revisited. Reumatologia 2014; 52: 351-353.

38. González-Gay MA, González-Juanatey C, Llorca J. Carotid ultrasound in the cardiovascular risk stratification of patients with rheumatoid arthritis: when and for whom? Ann Rheum Dis 2012; 71: 796-798.

39. Hippisley-Cox J, Coupland C, VinogradovaY, et al. Predicting cardiovascular risk in England and Wales: prospective derivation and validation of QRISK2. BMJ 2008; 336: 1475-1482.

40. Stone NJ, Robinson JG, Lichtenstein AH, et al. ACC/AHA Guideline on the Treatment of Blood Cholesterol to Reduce Atherosclerotic Cardiovascular Risk in Adults A Report of the American College of Cardiology/American Heart Association Task Force on Practice Guidelines. Circulation 2014; 129: 1-45.

41. Kavousi M, Leening MJ, Nanchen D, et al. Comparison of application of the ACC/AHA Guidelines, Adult Treatment Panel III guidelines, and European Society of Cardiology guidelines for cardiovascular disease prevention in a European cohort. JAMA 2014; 311: 1416-1423.

42. Tournadre A, Tatar Z, Pereira B, et al. Application of the European Society of Cardiology, Adult Treatment Panel III and American College of Cardiology/American Heart Association guidelines for cardiovascular risk management in a French cohort of rheumatoid arthritis. Int J Cardiol 2015; 183: 149-154.

43. Ridker PM, Cook NR. Statins: new American guidelines for prevention of cardiovascular disease. Lancet 2013; 382: 17621765.

44. Greenland P, Lauer MS. Cholesterol lowering in 2015 still answering questions about how and in whom. JAMA 2015; 314: 127-128.

45. Solomon DH, Greenberg J, Curtis JR, et al. Derivation and internal validation of an expanded cardiovascular risk prediction score for rheumatoid arthritis: a Consortium of Rheumatology Researchers of North America Registry Study. Arthritis Rheum 2015; 67: 1995-2003.

46. Ridker PM, Buring JE, Rifai N, et al. Development and validation of improved algorithms for the assessment of global cardiovascular risk in women the Reynolds Risk Score. JAMA 2007; 297: 611-619.

47. Ridker PM, Paynter NP, Rifai N, et al. C-reactive protein and parental history improve global cardiovascular risk prediction: the Reynolds Risk Score for men. Circulation 2008; 118: 22432251.

48. Pencina MJ, D’Agostino BD, Lason MG, et al. Predicting the 30Year Risk of Cardiovascular Disease The Framingham Heart Study. Circulation 2009; 119: 3078-3084.

49. Ralph B, D’Agostino S, Ramachandran S, et al. General cardiovascular risk profile for use in primary care the Framingham Heart Study. Circulation 2008; 117: 743-753.

50. Chung CP, Oeser A, Avalos I, et al. Utility of the Framingham risk score to predict the presence of coronary atherosclerosis in patients with rheumatoid arthritis. Arthritis Res Ther 2006; 8: R186. 
51. Mahoney LT, Burns TL, Stanford W, et al. Usefulness of the Framingham risk score and body mass index to predict early coronary artery calcium in young adults (Muscatine Study). Am J Cardiol 2001; 88: 509-515.

52. Chung CP, Oeser A, Avalos I, et al. Cardiovascular risk scores underestimate the presence of subclinical coronary-artery atherosclerosis in women with systemic lupus erythematosus. Lupus 2006; 15: 562-569.

53. Cullen P, Schulte H, Assmann G. The Münster Heart Study (PROCAM): total mortality in middle-aged men is increased at low total and LDL cholesterol concentrations in smokers but not in nonsmokers. Circulation 1997; 96: 2128-2136.

54. Assmann G, Cullen P, Schulte H. Simple scoring scheme for calculating the risk of acute coronary events based on the 10-year follow-up of the Prospective Cardiovascular Münster (PROCAM) Study. Circulation 2002; 105: 310-315.

55. Arts EEA. Transatlantic cardiovascular risk calculator for rheumatoid arthritis (ATACC-RA). Ann Rheum Dis 2014; 73: 123124.

56. Heslinga S, Van den Oever, I, Van Sijl A, et al. Cardiovascular risk management in patients with active Ankylosing Spondylitis: a detailed evaluation. BMC Musculoskeletal Disorders 2015; 16: 1-8.

57. Sattar N. How to assess the CVD risk in the individual patient? Ann Rheum Dis 2016 SP0012. DOI: 10.1136/annrheumdis-2016-eular.6435. 